\title{
Patient-specific Guides Using 3-dimensional Reconstruction Provide Accuracy and Reproducibility in Reverse Total Shoulder Arthroplasty
}

\author{
Jong Pil Yoon ${ }^{凶}$, Dong Hyun Kim, Jae Wook Jung, Chang-Hwa Lee, Seunggi Min, Hyun Joo Lee, Hee-June Kim \\ Department of Orthopaedic Surgery, Kyungpook National University Hospital, Daegu, Korea
}

\begin{abstract}
Background: We aimed to evaluate whether the use of our novel patient-specific guide (PSG) with 3-dimensional reconstruction in reverse total shoulder arthroplasty (RTSA) would allow accurate and reliable implantation of the glenoid and humeral components.

Methods: 20 fresh-frozen cadaveric shoulders were used. The PSG group $(n=10)$ and conventional group $(n=10)$ was evaluated the accuracy and reproducibility of implant positioning between before and after surgery on the computed tomography image.

Results: The superoinferior and anteroposterior offset in the glenoid component were $0.42 \pm 0.07,0.50 \pm 0.08$ in the conventional group and $0.45 \pm 0.03,0.46 \pm 0.02$ in the PSG group. The inclination and version angles were $-1.93^{\circ} \pm 4.31^{\circ}, 2.27^{\circ} \pm 5.91^{\circ}$ and $0.46^{\circ}$ $\pm 0.02^{\circ}, 3.38^{\circ} \pm 2.79^{\circ}$. The standard deviation showed a smaller difference in the PSG group. The anteroposterior and lateromedial humeral canal center offset in the humeral component were $0.45 \pm 0.12,0.48 \pm 0.15$ in the conventional group and $0.46 \pm 0.59$ $(p=0.794), 0.46 \pm 0.06(p=0.702)$ in the PSG group. The PSG showed significantly better humeral stem alignment.

Conclusions: The use of PSGs with 3-dimensional reconstruction reduces variabilities in glenoid and humerus component positions and prevents extreme positioning errors in RTSA.
\end{abstract}

(Clin Shoulder Ellbow 2019;22(1):16-23)

Key Words: Reverse total shoulder arthroplasty; Patient-specific guide; Glenoid component; Humeral component; Positioning

\section{Introduction}

Reverse total shoulder arthroplasty (RTSA) is known to improve the functional outcome of patients with cuff tear arthropathy. Most patients after RTSA report good clinical scores and pain reduction as well as recovery of range of motion. ${ }^{1,2)}$ In addition, owing to advances in surgical techniques and devices, decreasing complication rates are reported. ${ }^{3)}$

However, the RTSA procedure remains technically challenging and results in a number of complications such as scapular notching, early loosening, instability, and poor function. ${ }^{4,5)}$ In particular, these complications are associated with glenoid component malpositioning, especially superior tilt of the glenoid component. ${ }^{6}$ Variable scapular anatomy, glenoid bone loss, and joint stiffness contribute to difficulty in proper positioning of the glenoid component. ${ }^{7)}$ Abnormal glenoid morphology is observed in about $40 \%$ of patients requiring RTSA, making it unfeasible to place the glenoid component along the anatomic center line in these patients. ${ }^{8,9)}$

Preoperative planning with 3-dimensional (3D) imaging and patient-specific instrumentation (PSI) helps in resolving problems that might occur in total knee replacement, ${ }^{10,11)}$ and these techniques have recently been introduced in shoulder arthroplasty to overcome the problems of glenoid deformity and bone loss. Patient-specific guides (PSGs) have been suggested to improve the accuracy and reproducibility of the positioning of components in RTSA. ${ }^{7,12}$

Considering the above rationales, numerous studies have

Received August 15, 2018. Revised October 10, 2018. Accepted October 31, 2018.

Correspondence to: Jong Pil Yoon

Department of Orthopaedic Surgery, Kyungpook National University College of Medicine, 130 Dongdeok-ro, Jung-gu, Daegu 41944, Korea

Tel: +82-53-420-5628, Fax: +82-53-422-6605, E-mail: jpyoon@knu.ac.kr, ORCID: https://orcid.org/0000-0001-6446-6254

A cadaver study does not need an IRB approval.

Financial support: This work was supported by Institute for Information \& communications Technology Promotion (IITP) grant funded by the Korea government (MSIP) (\#B0101-14-1081). Conflict of interests: None. 
been initiated for PSG, particularly cadaver studies. ${ }^{7,13)}$ Although most patients with rotator cuff arthropathy have abnormalities of the glenoid, ${ }^{8,9)}$ cadavers with standardized glenoid (with no defect or deformity lesion) can be worked on, and has the advantage of allowing to check for actual reproducibility.

This study aimed to assess whether the use of our novel PSG, constructed using a 3D technique, in RTSA would allow accurate and reliable implantation of the glenoid and humeral components.

\section{Methods}

\section{Study Design}

Totally, 20 fresh-frozen cadaveric shoulders (male: 10, female: 10; mean age: 74.20 years) were utilized in this study. All cadavers had no shoulder pathology and bony deformity of the glenoid or humerus. All shoulders were scanned using computed tomography (CT) (Siemens SOMATOM Definition AS+; Siemens Belgium nv/SA, Beersel, Belgium) to plan the RTSA and design our novel glenoid and humeral components. The acquisition parameters were $120 \mathrm{~kW}$ and $224 \mathrm{Ma}$ (calculated based on the cadaveric anatomy); convolution kernel, B30f; slice thickness, $0.6 \mathrm{~mm}$; image size, $512 \times 512$; and field of view, $512 \times 0.44336$. The field of view of each scan included the entire scapula and humerus. A total of 20 specimens were randomly divided into the PSG group and the conventional group. The 10 cadavers in the PSG group were evaluated for entry point, angle, and position of guidewires after implantation with the PSG. The remaining 10 cadavers in the conventional group were guided and implanted without the PSG, and evaluated for

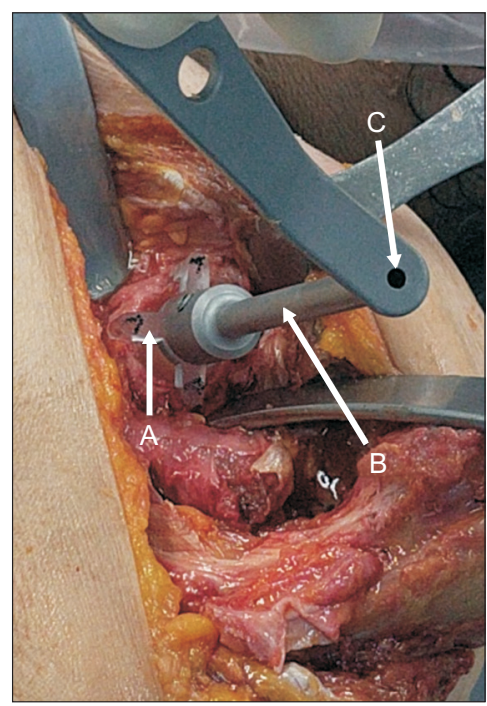

Fig. 1. Glenoid patient-specific guide. Glenoid attachment site (4-foot to fit well with the glenoid peripheral rim to prevent toggling) (A); metal handle attached to make it easy to operate (B); central hole with a short barrel to pass through the guide pin (C). the same outcomes stated above.

\section{Development of Our Novel Patient-specific Instrumentation Systems}

Korean government funding (Ministry of Science and Information \& Communication Technology) was provided for manufacturing our novel PSG systems. The glenoid PSG was designed to allow the surgeon to introduce it onto the glenoid to prevent toggling; it had 4 supporting bars at attachment site with the glenoid peripheral rim to fit well. A central hole with a short barrel through which the guide pin could pass was created, and a metal handle was attached to make it easy to operate (Fig. 1). The humeral PSG was designed to exactly pass the center of the humeral intramedullary canal. It was made to fit well on the articular surface and bicipital groove, and a cutting guide was assembled for use (Fig. 2).

\section{Preoperative Planning (Glenoid and Humeral Surgical Guides)}

The dedicated software Coralis reference PSI system (Corentec, Seoul, Korea) was used to create 3D surface models of all scapula and humerus based on the obtained CT images. The glenoid PSG was designed based on a scapular plane defined by 3 points (glenoid center point, scapula trigonum, and inferior angle of the scapula), as described in a previous study. ${ }^{12)}$ The guide entry was the center of the glenoid and was directed towards the neutral tilt. The humeral PSG was designed to allow the central guide to enter the long axis of the humeral medulla. The guide was designed to allow neck cutting with the cadaver's own articular portions and with inherent humeral retroversion (Fig. 2). The designed PSG was printed with natural polyamide PA2200 resin (EOSINT P380 selective laser sintering machine; EOS GmbH, Krailling, Germany).

\section{Cadaveric Surgical Procedure}

All procedures were performed by a single experimental surgeon, with the cadaver specimens placed in the supine position.
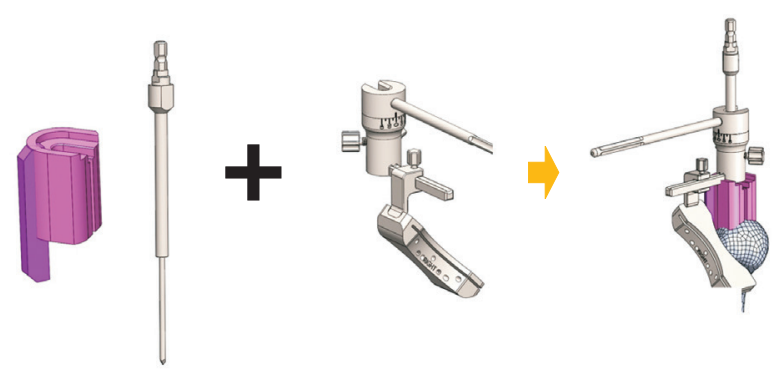

Fig. 2. Humeral patient-specific guide was designed to exactly pass the center of the humeral intramedullary canal. It was made to fit well on the articular surface and bicipital groove, and a cutting guide was assembled for humeral neck cutting. 
Through the delto-pectoral approach, the glenoid and humeral heads were exposed with minimal incision in the conjoined tendon, and by cutting the long head of the biceps tendon and subscapularis tendon. The remaining glenoid labrum was removed, releasing the head of the triceps. The rotator cuff was resected to mimic a cuff tear arthropathy to be further subjected to RTSA.

This study was divided into 2 methods. The first study concerned inserting only the guidewire; the glenoid and humeral guidewires (2.6 mm Steinman pin) were inserted, and humerus neck cutting was performed according to the predesigned and 3D printed PSG in the PSG group. In the conventional group, the glenoid entry point was determined according to our standard methods, based on the intraoperative interpretation of the scapular position and glenoid morphology, center of glenoid fossa, and neutral tilt. The insertion point of the humerus was defined using the freehand technique based on the long axis of the humeral medulla. Neck cutting was performed after insertion of the guidewire (Fig. 3).

In the second study of implant insertion, humeral neck cutting was performed after inserting the guide using the PSC or the conventional method. Glenoid reaming was performed along the guidewire, and the baseplate was positioned and fixed using central, superior, and inferior screws. The hemispheres were fixed to the baseplate through the press-fit method. This was followed by humeral medullary reaming, and the humeral stem was inserted. After fixing the liner and plate on the humeral stem, the joint was reduced and the operation was completed.

\section{Radiologic Evaluation}

Postoperative CT scan was conducted to determine the glenoid and humeral component positioning, and differences from the preoperatively planned implant were calculated. Postoperative CT scan was performed in the same manner as the preoperative $\mathrm{CT}$ scan.

The following radiological measurements of the glenoid were obtained:

(1) Superoinferior offset (distance ratio between the center of the guidewire or implants and the glenoid superior or inferior margin in coronal planes) (Fig. 4)

(2) Anteroposterior offset (distance ratio between the center of the guidewire or implants and the glenoid anterior or posterior margin in axial planes)

(3) Inclination (angle between the implant axis defined by the center peg of the implant and the line joining the superior and inferior margins of the glenoid, as described by Maurer et al. ${ }^{14}$ )

(4) Version (angle between the implant axis and the scapula axis defined by the line connecting the medial end of the scapula and the midpoint of the glenoid fossa, as described by Friedman et al. ${ }^{15}$ )

The following radiological measurements of the humerus were obtained:

(5) Humeral stem-canal center offset (anteroposterior) in the axial plane (actual stem location [anteroposterior] compared with the humeral canal center axis in the axial plane)

(6) Humeral stem-canal center offset (lateromedial) in the

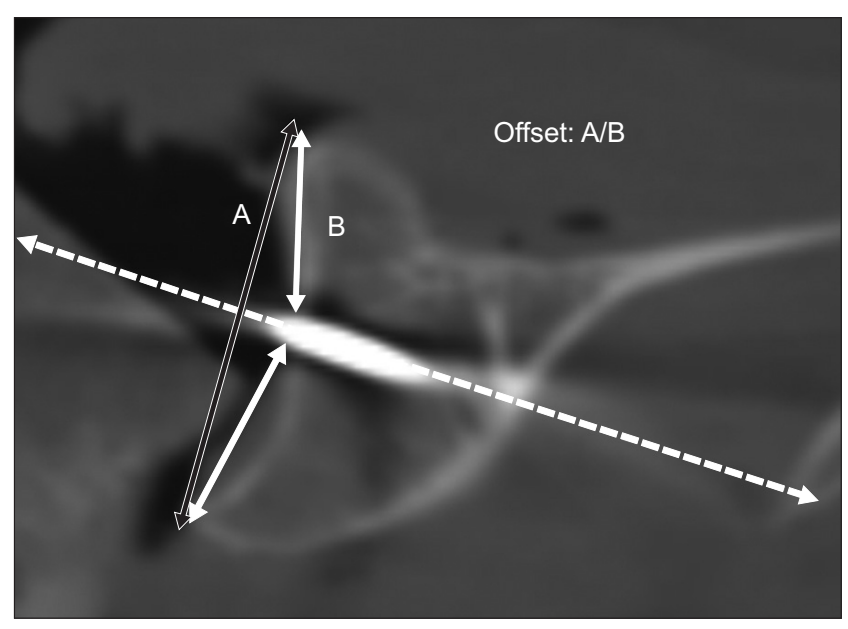

Fig. 4. Superoinferior offset defined distance between the center of the guidewire or implants (B) and the glenoid superior or inferior margin in coronal planes (A).
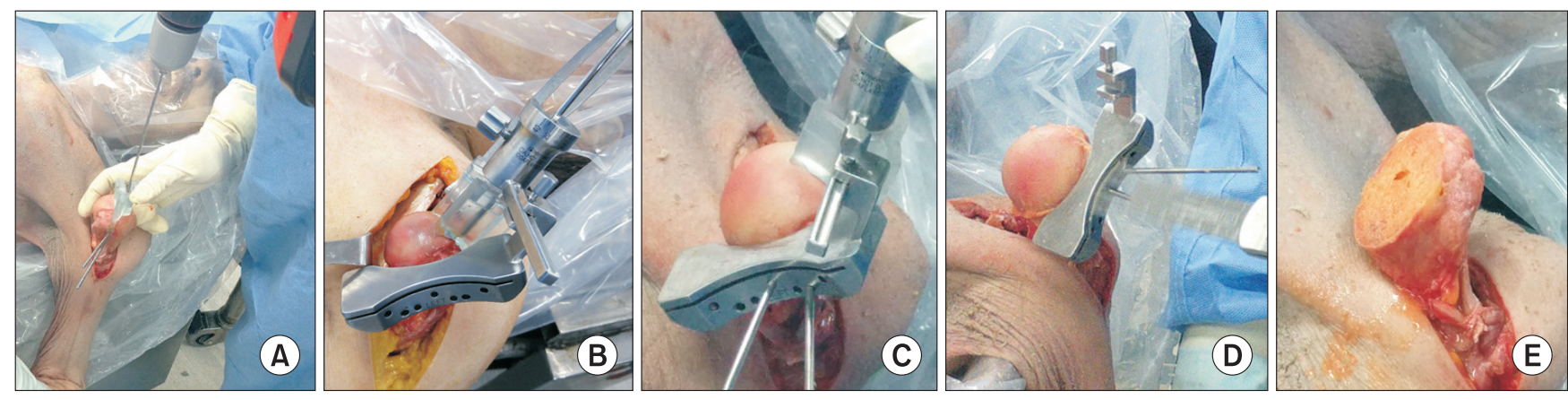

Fig. 3. Flow of surgical procedure: (A) Fix the humeral zig to the humeral head and insert the humeral guidewire. (B, C) Combine the humeral patient-specific instrumentation zig with the cutting guide and fix the cutting guide with the Steinman pin. (D) Leave only the cutting guide and remove the rest. (E) Finally, perform humerus neck cutting according to the predesigned patient-specific guide. 
axial plane (actual stem location [lateromedial] compared with the humeral canal center axis in the axial plane) (Fig. 5A)

(7) Humeral stem-canal center angle in the coronal plane (angle difference of the stem axis with the long axis of the humeral medulla) (Fig. 5B)

In addition, the preoperative and postoperative reconstructed images were compared for the position and angle before and after surgery. The 3D models from the preoperative planning were registered to the postoperative $3 \mathrm{D}$ models by using the iterative closest point registration algorithm. ${ }^{16)}$ Along with the guidewire indicating the position of the implant, this enabled us to determine the deviation between the planned position of the implant and the postoperatively achieved position.

\section{Statistical Analysis}

The mean values were compared using Student's t-test or the Mann-Whitney U-test for continuous variables, and the chisquare or Fisher's exact tests for categorical variables, in order to statistically evaluate the differences between the 2 groups. Sta-

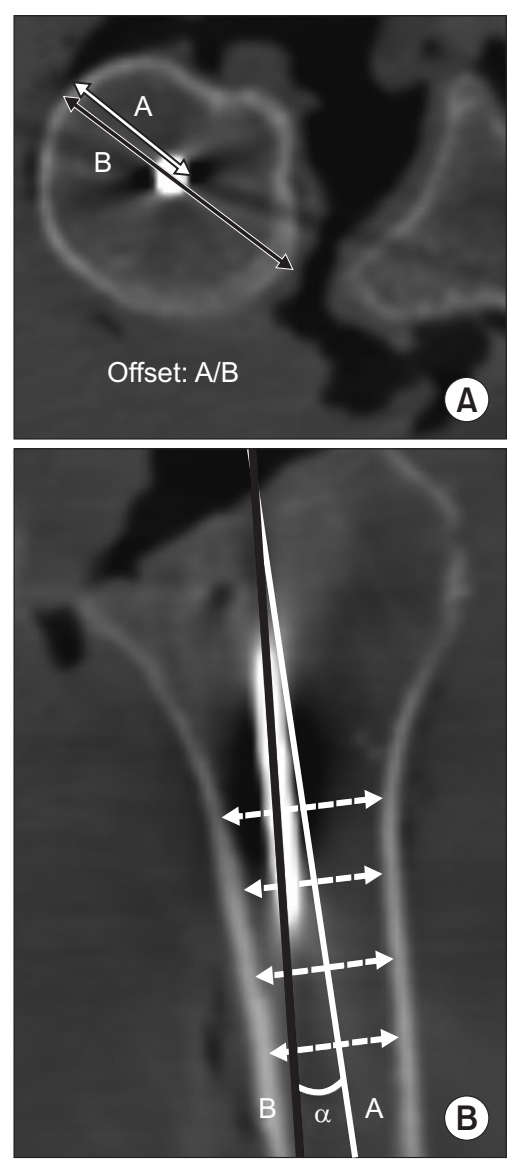

Fig. 5. (A) Humeral stem canal center offset in the axial plane. Line A is distance between the center of the guidewire or implant and the lateral or medial margin of the humerus in the axial planes; Line B is distance between the humerus lateral margin and the humerus medial margin in the axial planes. (B) Humeral stem canal center angle $(\alpha)$ in the coronal plane: Measured angle difference of the stem axis (line B) with the long axis of humeral medulla (line A). tistical analyses were conducted using the SPSS version 17.0 for Windows (SPSS Inc., Chicago, IL, USA). All statistical tests were 2 -tailed, and a $p$-value of $<0.05$ was considered statistically significant.

\section{Results}

\section{Baseline Comparison}

A total of 20 cadavers were divided into 2 groups, comprising of 10 cadavers each: the conventional group and the PSG group. Each group included 5 female and 5 male cadavers $(p>0.999)$. Following results were obtained on comparing the various parameters between the conventional group and the PSG group ( \pm standard deviation [SD]): mean age $74.20 \pm 8.25$ years and $74.20 \pm 8.25$ years, respectively, $(p>0.999)$; glenoid width $26.49 \pm 2.52 \mathrm{~mm}$ and $26.66 \pm 2.49 \mathrm{~mm}$, respectively, $(p=0.727)$; and glenoid height $32.80 \pm 2.00 \mathrm{~mm}$ and 33.05 $\pm 2.19 \mathrm{~mm}$, respectively, $(p=0.788)$. Overall, the baseline data showed no significant difference between both groups (Table 1).

\section{Glenoid Alignment}

Using the 3D planning automatic software, CT scans measured the preoperative and postoperative version and inclination angles. We observed that PSG significantly reduced deviations from the planned glenoid implant positioning. Comparing the conventional and PSG groups, the superoinferior offset in the alignment of the glenoid component was $0.42 \pm 0.07$ and 0.45 \pm 0.03 , respectively; the anteroposterior offset was $0.50 \pm$ 0.08 and $0.46 \pm 0.02$, respectively; the inclination angle was $-1.93^{\circ} \pm 4.31^{\circ}$ and $-1.97^{\circ} \pm 3.58^{\circ}$, respectively; and the version angle was $2.27^{\circ} \pm 5.91^{\circ}$ and $3.38^{\circ} \pm 2.79^{\circ}$, respectively (Table 2). The mean value and distribution of each alignment value (superoinferior offset, anteroposterior offset, version, and inclination) of both groups were statistically not different (all $p>0.05$ ); however, the SD differed to a smaller extent in the PSG group (Fig. 6).

\section{Humeral Alignment}

Considering measurements of the humeral alignment, the following values were obtained for the conventional and PSG

Table 1. Demographic and Clinical Data

\begin{tabular}{lccc}
\multicolumn{1}{c}{ Variable } & Conventional group & PSG group & p-value \\
\hline Number & 10 & 10 & \\
Sex (male:female) & $5: 5$ & $5: 5$ & $>0.999$ \\
\hline Age (yr) & $74.20 \pm 8.25$ & $74.20 \pm 8.25$ & $>0.999$ \\
Glenoid width (mm) & $26.49 \pm 2.52$ & $26.66 \pm 2.49$ & 0.727 \\
\hline Glenoid height $(\mathrm{mm})$ & $32.80 \pm 2.00$ & $33.05 \pm 2.19$ & 0.788 \\
\hline
\end{tabular}

Values are presented as number only or mean \pm standard deviation. PSG: patient-specific guide. 
groups: the anteroposterior humeral canal center offset in the humeral alignment was $0.45 \pm 0.12$ and $0.46 \pm 0.59$, respectively $(p=0.794)$; the lateromedial humeral canal center offset was $0.48 \pm 0.15$ and $0.46 \pm 0.06$, respectively $(p=0.702)$; and the humeral stem canal center angle was $4.98^{\circ} \pm 2.31^{\circ}$ and $2.64^{\circ} \pm 1.07^{\circ}$, respectively $(p=0.009)$. These results indicate that the PSG group had significantly better humeral stem alignment (Table 3).

Table 2. Alignment of Glenoid Component

\begin{tabular}{lccc}
\hline \multicolumn{1}{c}{ Variable } & Conventional group & PSG group & $p$-value \\
\hline Number & 10 & 10 & \\
Superoinferior offset & $0.42 \pm 0.07$ & $0.45 \pm 0.03$ & 0.339 \\
Anteroposterior offset & $0.50 \pm 0.08$ & $0.46 \pm 0.02$ & 0.146 \\
Inclination $\left(^{\circ}\right)$ & $-1.93 \pm 4.31$ & $-1.97 \pm 3.58$ & 0.983 \\
Version $\left(^{\circ}\right)$ & $2.27 \pm 5.91$ & $3.38 \pm 2.79$ & 0.600
\end{tabular}

Values are presented as number only or mean \pm standard deviation.

PSG, patient-specific guide.
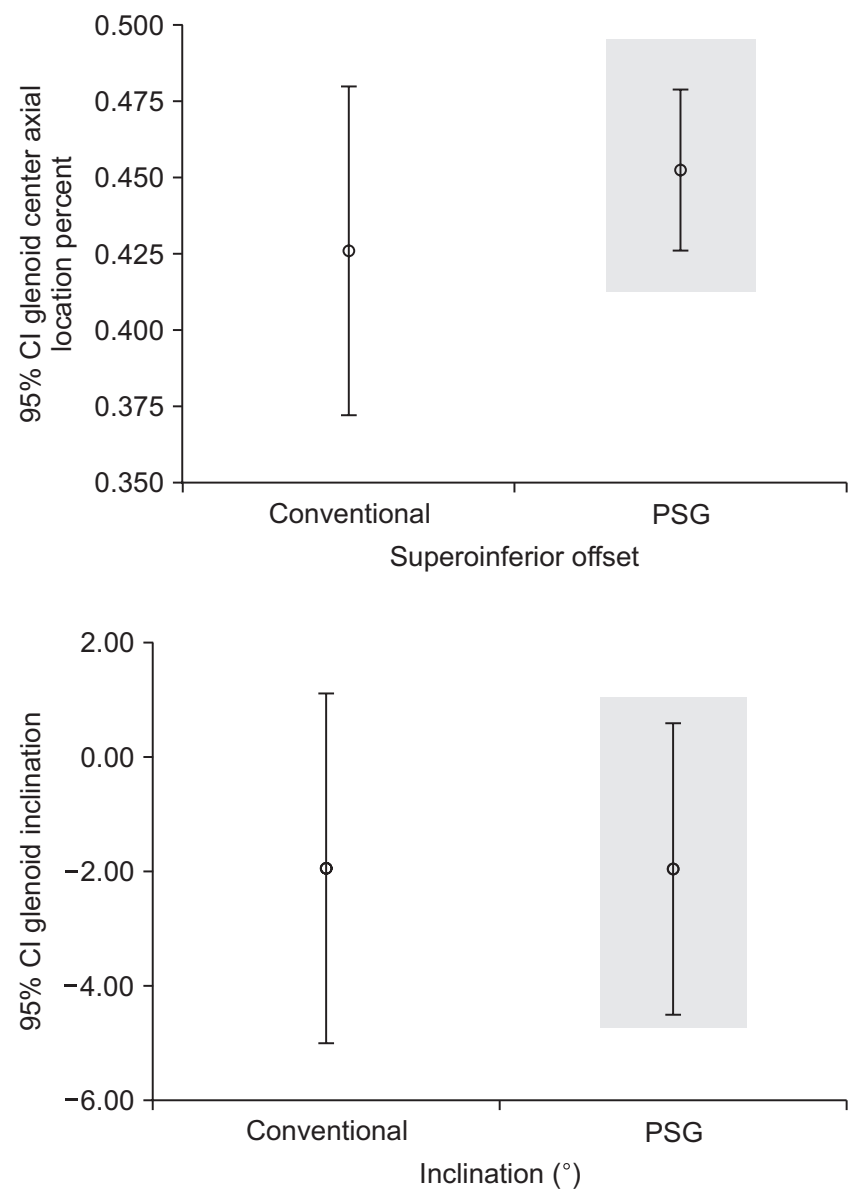

\section{Discussion}

This study is a prospective randomized clinical trial designed to test the hypothesis that applying PSGs with 3D reconstruction technique is superior to conventional surgical techniques for the placement of glenoid and humerus components in cadaveric shoulders. The SDs of superoinferior offset, anteroposterior offset, inclination, and version were lower in the PSG group than in

Table 3. Alignment of Humeral Component

\begin{tabular}{lccc}
\hline \multicolumn{1}{c}{ Variable } & Conventional & PSG & -value \\
\hline Number & 10 & 10 & \\
$\begin{array}{c}\text { Humeral stem canal center } \\
\text { offset (anteroposterior) }\end{array}$ & $0.45 \pm 0.12$ & $0.46 \pm 0.59$ & 0.794 \\
$\begin{array}{c}\text { Humeral stem canal center } \\
\text { offset (lateromedial) }\end{array}$ & $0.48 \pm 0.15$ & $0.46 \pm 0.06$ & 0.702 \\
$\begin{array}{c}\text { Humeral stem canal center } \\
\text { angle }\left(^{\circ}\right)\end{array}$ & $4.98 \pm 2.31$ & $2.64 \pm 1.07$ & 0.009 \\
\hline
\end{tabular}

Values are presented as number only or mean \pm standard deviation.

PSG: patient-specific guide.
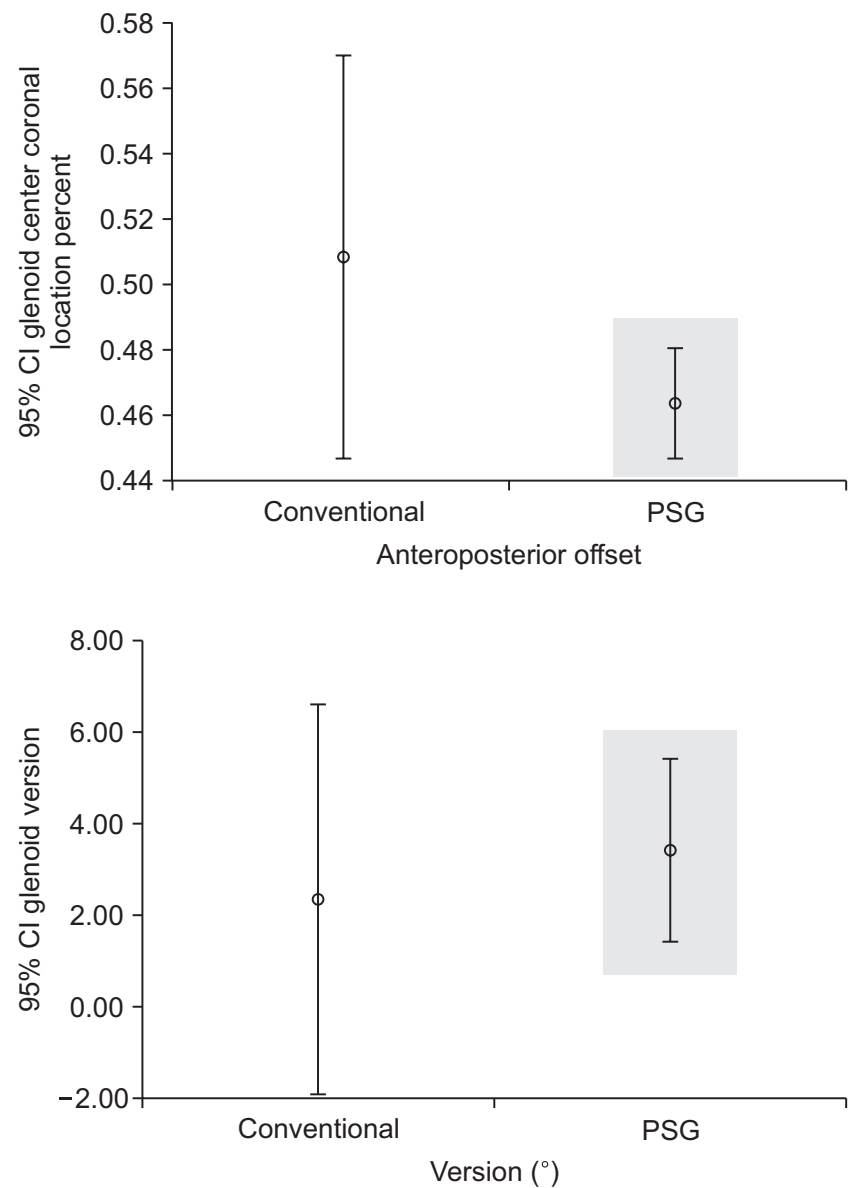

Fig. 6. The comparison of glenoid alignment. There were no significant differences between superoinferior offset, anteroposterior offset, version, and inclination between groups; however, the standard deviation showed a smaller difference in the patient-specific guide (PSG).

CI: confidence interval. 
the conventional group, indicating that the use of PSG is more consistent than the conventional method in positioning the glenoid component. Furthermore, the humeral stem canal center angle in the humeral component was significantly lower in the PSG group $(p=0.009)$. The humeral stem in the PSG group was precisely located at the center of the medullary canal of the humerus, compared with the conventional group.

The RTSA procedure is technically challenging and is reported to be associated with instability, early loosening, scapular notching, and poor function, especially due to glenoid component malpositioning. ${ }^{4,5,17-19)}$ Errors in inclination angle are known to impart the greatest probability for clinical failure in total shoulder arthroplasty (TSA) ${ }^{20)}$ Similarly, in RTSA, errors in inclination need to be avoided due to increased scapular notching and component loosening. ${ }^{21)}$

However, it is more difficult to control the version and slope, especially if the original articular surface is not aligned neutrally owing to wear or abnormal shape; thus, accuracy and reproducibility are dependent on the surgeon's skills and interpretation of the position and shape of the scapula.

To solve these problems, some authors used computerassisted navigation to improve the accuracy of glenoid component positioning and wear correction. ${ }^{22,23)}$ However, navigated systems often require large and expensive equipment for implementation, thereby increasing the operating time by $20 \%$, and conferring additional cost to the procedure..$^{24,25)}$

As an alternative method to overcome this problem, PSGs created using the 3D reconstruction technique enable preoperative planning with automatic software. Moreover, the use of PSC provides accurate and reproducible positioning and orientation of the glenoid and humeral components in RTSA. Early cadaver studies on TSA and RTSA have reported the accuracy and reproducibility of PSI. Levy et al. ${ }^{7)}$ reported the accuracy of PSI in positioning the glenoid baseplate in RTSA. They reported that the translational accuracy of the starting point for the guide pin averaged $1.2 \pm 0.7 \mathrm{~mm}$, whereas the accuracy of the inferior inclination was $1.2^{\circ} \pm 1.2^{\circ}$ and that of the glenoid version was $2.6^{\circ} \pm 1.7^{\circ}$. Their study concluded that PSGs were highly accurate in reproducing the starting point, inclination, and version set on preoperative guides. Walch et al. ${ }^{26)}$ also showed a good correlation between preoperative planning and the achieved position of the guide pin in an in vitro study on the use of PSI for glenoid component placement in TSA. They reported a mean entry point position error of $1.05 \pm 0.31 \mathrm{~mm}$, a mean inclination error of $1.42^{\circ} \pm 1.37^{\circ}$, and a mean version error of $1.64^{\circ} \pm$ $1.01^{\circ}$.

In an in vivo study, Gauci et al. ${ }^{27)}$ reported that the mean error of the glenoid component in PSI was lower than that of the glenoid component and the preoperative planning value in anatomical TSA. The mean error in the accuracy of the entry point was $-0.1 \pm 1.4 \mathrm{~mm}$ in the horizontal plane and $0.8 \pm 1.3 \mathrm{~mm}$ in the vertical plane. The mean error in the orientation of the glenoid component was $3.4^{\circ} \pm 5.1^{\circ}$ for version and $1.8^{\circ} \pm 5.3^{\circ}$ for inclination. In a series of 10 consecutive patients who underwent TSA and RTSA with PSI, Suero et al. ${ }^{25)}$ also reported that PSI enables adjusting the position of the glenoid component and screws.

However, in a recent study, Lau and Keith ${ }^{28)}$ claimed that PSI was not as successful as it appeared in previous studies. They produced PSGs that permitted $0^{\circ}$ glenoid inclination and version in TSA, and $10^{\circ}$ inferior inclination in RTSA. For the TSA group, the mean version was measured at $8^{\circ} \pm 10^{\circ}$ retroversion and $1^{\circ}$ $\pm 4^{\circ}$ inclination. For the RTSA group, the mean version was $10^{\circ}$ $\pm 10^{\circ}$ retroversion and $-1^{\circ} \pm 5^{\circ}$ inclination. They demonstrated that the use of PSGs does not automatically allow flawless glenoid component positioning. We designed PSGs for accurate implant positioning by applying 3D CT and automatic software, and showed good consistency in cadavers. However, it is unknown whether our PSG will show good results in vivo.

The most important aspect in the implantation of a humeral component in shoulder arthroplasty is to set the correct entry point at the center of the humeral canal. Failure to set the correct entry point results in malalignment, such as varus of the humeral component, which in turn leads to long-term implant failure. Another important aspect of the humeral component is that neck cutting should be precise and should match the version and inclination of the patient's own humeral anatomical neck. If the retroversion becomes larger or smaller, the external rotation and internal rotation may be restricted. ${ }^{29,30)}$ As per our understanding, there are few reports on implantation of humeral components using PSG. We designed a humeral PSG to improve the humeral stem alignment, and our results indicate better stem alignment than the conventional method. However, the PSG system only provides a helpful anatomical guideline of the humeral canal for surgeons, and does not replace the comprehensive understanding of the complex anatomy of the proximal humeral.

The current study is meaningful in being a level I clinical trial using cadavers to evaluate this technique in RTSA. However, there are some limitations to our technique and study, which are consistent with the precautions required when applying PSI in RTSA. First, creating a PSG depends on a precisely defined preoperative plan using the accurate 3D model of the patient's scapula. Although accurate 3D models of the scapula and humerus need to be separated from the surrounding soft tissue, this could prove difficult owing to cartilage loss, severe bone defect, calcification, stiffness, and previous fractures. Second, a stable fit and precise positioning of the PSI guide to the glenoid rim is dependent on exposure after sufficient removal of the soft tissue around the joint surface; thus, there is a possibility of damage to important structures and subsequent complications. Third, this study lacks analysis of other variables such as screw position. The 
guidelines for glenoid positioning, which consider the peripheral screws used and the patient's bone quality, are currently unclear. In this study, version and tilt were used as neutral values, but this was not true for all cadavers. More research is required to investigate the ideal glenoid positioning for maximum functionality and mechanical stability.

\section{Conclusion}

Compared to the standard conventional surgical techniques, the use of PSGs with a 3D reconstruction technique reduces variability in the positioning of glenoid and humerus components and prevents extreme position errors in RTSA. However, although application of PSG adds to the preoperative planning time, the surgeon's intuition remains important. The clinical effects, costs, and long-term effects of this technology require further cost-benefit analysis and clinical research.

\section{References}

1. Cazeneuve JF, Cristofari DJ. Long term functional outcome following reverse shoulder arthroplasty in the elderly. Orthop Traumatol Surg Res. 2011;97(6):583-9. doi: 10.1016/ j.otsr.2011.03.025.

2. Sirveaux F, Favard L, Oudet D, Huquet D, Walch G, Molé D. Grammont inverted total shoulder arthroplasty in the treatment of glenohumeral osteoarthritis with massive rupture of the cuff. Results of a multicentre study of 80 shoulders. J Bone Joint Surg Br. 2004;86(3):388-95. doi: 10.1302/0301-620X.86B3.14024.

3. Bohsali KI, Bois AJ, Wirth MA. Complications of shoulder arthroplasty. J Bone Joint Surg Am. 2017;99(3):256-69. doi: 10.2106/JBJS.16.00935.

4. Boileau P, Watkinson DJ, Hatzidakis AM, Balg F. Grammont reverse prosthesis: design, rationale, and biomechanics. J Shoulder Elbow Surg. 2005;14(1 Suppl S):147S-61S. doi: 10.1016/ j.jse.2004.10.006.

5. Chalmers PN, Rahman Z, Romeo AA, Nicholson GP. Early dislocation after reverse total shoulder arthroplasty. J Shoulder Elbow Surg. 2014;23(5):737-44. doi: 10.1016/j.jse.2013.08.015.

6. Gutiérrez S, Walker M, Willis M, Pupello DR, Frankle MA. Effects of tilt and glenosphere eccentricity on baseplate/bone interface forces in a computational model, validated by a mechanical model, of reverse shoulder arthroplasty. J Shoulder Elbow Surg. 2011;20(5):732-9. doi: 10.1016/j.jse.2010.10.035.

7. Levy JC, Everding NG, Frankle MA, Keppler LJ. Accuracy of patient-specific guided glenoid baseplate positioning for reverse shoulder arthroplasty. J Shoulder Elbow Surg. 2014; 23(10):1563-7. doi: 10.1016/j.jse.2014.01.051.

8. Barrett WP, Franklin JL, Jackins SE, Wyss CR, Matsen FA 3rd. Total shoulder arthroplasty. J Bone Joint Surg Am. 1987;69(6):
865-72.

9. Frankle MA, Teramoto A, Luo ZP, Levy JC, Pupello D. Glenoid morphology in reverse shoulder arthroplasty: classification and surgical implications. J Shoulder Elbow Surg. 2009;18(6):87485. doi: 10.1016/j.jse.2009.02.013.

10. Ng VY, DeClaire JH, Berend KR, Gulick BC, Lombardi AV Jr. Improved accuracy of alignment with patient-specific positioning guides compared with manual instrumentation in TKA. Clin Orthop Relat Res. 2012;470(1):99-107. doi: 10.1007/ s11999-011-1996-6.

11. Victor J, Premanathan A. Virtual 3D planning and patient specific surgical guides for osteotomies around the knee: a feasibility and proof-of-concept study. Bone Joint J. 2013;95(11 Suppl A):153-8. doi: 10.1302/0301-620X.95B11.32950.

12. Hendel MD, Bryan JA, Barsoum WK, et al. Comparison of patient-specific instruments with standard surgical instruments in determining glenoid component position: a randomized prospective clinical trial. J Bone Joint Surg Am. 2012;94(23):216775. doi: 10.2106/JBJS.K.01209.

13. Throckmorton TW, Gulotta LV, Bonnarens FO, et al. Patientspecific targeting guides compared with traditional instrumentation for glenoid component placement in shoulder arthroplasty: a multi-surgeon study in 70 arthritic cadaver specimens. J Shoulder Elbow Surg. 2015;24(6):965-71. doi: 10.1016/j.jse.2014.10.013.

14. Maurer A, Fucentese SF, Pfirrmann CW, et al. Assessment of glenoid inclination on routine clinical radiographs and computed tomography examinations of the shoulder. J Shoulder Elbow Surg. 2012;21(8):1096-103. doi: 10.1016/ j.jse.2011.07.010.

15. Friedman RJ, Hawthorne KB, Genez BM. The use of computerized tomography in the measurement of glenoid version. J Bone Joint Surg Am. 1992;74(7):1032-7.

16. Besl PJ, McKay ND. A method for registration of 3-D shapes. TPAMI. 1992;14(2):239-56. doi: 10.1109/34.121791.

17. Harman M, Frankle M, Vasey M, Banks S. Initial glenoid component fixation in "reverse" total shoulder arthroplasty: a biomechanical evaluation. J Shoulder Elbow Surg. 2005;14(1 Suppl S):162S-7S. doi: 10.1016/j.jse.2004.09.030.

18. Crosby LA, Hamilton A, Twiss T. Scapula fractures after reverse total shoulder arthroplasty: classification and treatment. Clin Orthop Relat Res. 2011;469(9):2544-9. doi: 10.1007/s11999011-1881-3.

19. Virani NA, Harman M, Li K, Levy J, Pupello DR, Frankle MA. In vitro and finite element analysis of glenoid bone/baseplate interaction in the reverse shoulder design. J Shoulder Elbow Surg. 2008;17(3):509-21. doi: 10.1016/j.jse.2007.11.003.

20. Hopkins AR, Hansen UN, Amis AA, Emery R. The effects of glenoid component alignment variations on cement mantle stresses in total shoulder arthroplasty. J Shoulder Elbow Surg. 2004;13(6):668-75. doi: 10.1016/S1058274604001399. 
21. Gerber C, Pennington SD, Nyffeler RW. Reverse total shoulder arthroplasty. J Am Acad Orthop Surg. 2009;17(5):284-95.

22. Edwards TB, Gartsman GM, O'Connor DP, Sarin VK. Safety and utility of computer-aided shoulder arthroplasty. J Shoulder Elbow Surg. 2008;17(3):503-8. doi: 10.1016/ j.jse.2007.10.005.

23. Verborgt O, De Smedt T, Vanhees M, Clockaerts S, Parizel PM, Van Glabbeek F. Accuracy of placement of the glenoid component in reversed shoulder arthroplasty with and without navigation. J Shoulder Elbow Surg. 2011;20(1):21-6. doi: 10.1016/j.jse.2010.07.014.

24. Kircher J, Wiedemann M, Magosch P, Lichtenberg S, Habermeyer P. Improved accuracy of glenoid positioning in total shoulder arthroplasty with intraoperative navigation: a prospective-randomized clinical study. J Shoulder Elbow Surg. 2009;18(4):515-20. doi: 10.1016/j.jse.2009.03.014.

25. Suero EM, Citak M, Lo D, Krych AJ, Craig EV, Pearle AD. Use of a custom alignment guide to improve glenoid component position in total shoulder arthroplasty. Knee Surg Sports Traumatol Arthrosc. 2013;21(12):2860-6. doi: 10.1007/s00167012-2177-1.
26. Walch G, Vezeridis PS, Boileau P, Deransart P, Chaoui J. Threedimensional planning and use of patient-specific guides improve glenoid component position: an in vitro study. J Shoulder Elbow Surg. 2015;24(2):302-9. doi: 10.1016/ j.jse.2014.05.029.

27. Gauci MO, Boileau P, Baba M, Chaoui J, Walch G. Patientspecific glenoid guides provide accuracy and reproducibility in total shoulder arthroplasty. Bone Joint J. 2016;98(8):1080-5. doi: 10.1302/0301-620X.98B8.37257.

28. Lau SC, Keith PPA. Patient-specific instrumentation for total shoulder arthroplasty: not as accurate as it would seem. J Shoulder Elbow Surg. 2018;27(1):90-5. doi: 10.1016/j.jse. 2017.07.004.

29. Gulotta LV, Choi D, Marinello P, et al. Humeral component retroversion in reverse total shoulder arthroplasty: a biomechanical study. J Shoulder Elbow Surg. 2012;21(9):1121-7. doi: 10.1016/j.jse.2011.07.027.

30. Li X, Knutson Z, Choi D, et al. Effects of glenosphere positioning on impingement-free internal and external rotation after reverse total shoulder arthroplasty. J Shoulder Elbow Surg. 2013;22(6):807-13. doi: 10.1016/j.jse.2012.07.013. 